\title{
Immunization, surveillance, and the global health security agenda: historical perspectives and implications for national immunization programs
}

\author{
Imunização, vigilância e a agenda global de \\ segurança sanitária: perspectivas históricas \\ e implicações para programas nacionais \\ de imunização
}

Inmunización, vigilancia, y agenda global de la seguridad de la salud: perspectivas históricas e implicaciones para los programas nacionales de inmunización

Jon Kim Andrus 1

doi: 10.1590/0102-311X00123819

\section{Background}

Unlike most other disease prevention programs in public health, the ones involving vaccine-preventable diseases through well-managed national immunization programs (NIPs) have benefited by rapidly expanding science of vaccinology 1 . The prime objective of NIPs remains as: to administer the available, safe, and effective vaccines to all, especially to those that are most at risk. By prioritizing vulnerable populations - hard-to-reach rural populations, poor urban slums, migratory populations, pregnant women, and chronically underserved indigenous communities - NIPs become important drivers of reducing inequities in health, thus saving more lives more quickly, administering the benefits of the traditional and now the newer vaccines 2 .

For such purpose, surveillance is critically important, since knowing where disease occurs is the best approach for determining the communities that are most at risk, to receive life-saving technologies. Infectious diseases respect no borders; they are only a plane ride away ${ }^{3}$. An ideal, inclusive and regional approach to prevent and control vaccine-preventable diseases (VPDs) provides effective coordination and technical cooperation across borders, regardless of political ideologies of neighboring countries 4 . Health security among all communities and among all countries supersedes political differences. Among many factors, VPD surveillance again is a key factor; additionally, accurate vaccination coverage data also aids with the prioritization of underserved areas.

As we move closer to a world without polio, successfully sustaining the assets of the global eradication initiative (GPEI) will require the prioritization of comprehensive VPD surveillance as a global good 5. Strengthening VPD surveillance in many countries, especially those of the Latin America and the Caribbean and Southeast Asia, resulted from building upon the polio foundation to implement a greatly expanded rash and fever surveillance system used for measles-rubella elimination. The foundation of polio surveillance, reinforced with rash and fever surveillance, in turn, becomes the backbone of all comprehensive VPD surveillance. Global health security should logically benefit from high-quality VPD disease surveillance and vaccination coverage, supported by accountable international health regulations (IHR) 6 . The aim of this article is to share personal insights on how the work towards implementing comprehensive VPD surveillance combined with high-quality vaccination responses may provide lessons for maintaining global health security. 


\section{Brief history of polio eradication and the role of surveillance}

With unrelenting implementation of the polio eradication strategies - routine immunization, national immunization days, mopping-up vaccination campaigns, and high-quality surveillance of acute flaccid paralysis (AFP) with technical back-up and oversight of Pan American Health Organization (PAHO) - the last case of polio in the Americas occurred in Peru on August 23, 1991, only 8 months and 23 days past the intended target of 1990 7. This experience emphasized the importance of highlevel political commitment, as well as technical and operational excellence, with a doggedly persistent vision adherent to agreed-upon targets. The program was prioritized as time-bound, with an almost maniacal sense of duty and work ethic. Management and supervision were almost always field-based, with a skeletal staff at the PAHO Head Quarters.

Technical and operational excellence was best exemplified by the diligence and effort that went into sustaining high-quality surveillance ${ }^{3}$. This work was inextricably linked to a laboratory network, which was also grounded in research and innovation. Surveillance data drove targeted mopping-up campaigns serving as the final blow to interrupt wild poliovirus transmission. Surveillance data were also used to modify the polio case definitions and other surveillance and vaccination strategies 8 . Innovation was most apparent in the development of laboratory technologies that were transferred to all labs in the network, and an operational research agenda, such as environmental surveillance of wild poliovirus 9 .

Polio was deeply embedded into the national plans of action of NIPs, never separated from essential immunization services, largely due to the leadership and vision of Dr. Ciro de Quadros 10. Thus, routine immunization coverage levels remained high, and trivalent oral polio vaccine was the vaccine of choice until the very end. Since routine immunization was always prioritized, the legacy of polio eradication in the Americas immediately fed into the efforts to successful eliminate neonatal tetanus, introduce new vaccines, and ultimately embark on the elimination of measles, rubella, and congenital rubella syndrome (CRS).

\section{Progress towards measles and rubella elimination}

With a similar approach to unrelenting implementation of the measles-rubella elimination strategies - sustaining high levels of routine coverage of measles-rubella (MR) containing vaccine, catch-up campaigns, follow-up vaccination campaigns, measles-rubella elimination vaccination campaigns targeting an expanded age range, and high-quality expanded rash and fever surveillance with extensive laboratory support - interruption of endemic transmission of measles occurred in 2002, followed by rubella in 2009 11. Rubella elimination came after measles, because the implementation of the expanded, age-range campaigns, in most countries for all women and men aged $<40$ years, were conducted later. A key lesson from all the efforts was that with high routine MR containing vaccine coverage, only one expanded, age-range rubella elimination campaign was required to stop rubella virus transmission, and ultimately eliminate the devastating sequences of CRS.

Unfortunately, the program has been confronted with a number of setbacks, all stemming from the constant threat of viral importations from the rest of the world 12. Currently, the question for PAHO member states is not "if" importations from other parts of the world will occur, but rather "when" they will occur. Countries invariably receive importations, and if they can stop transmission related to such importations within one year, they can then maintain their measles-rubella free status. However, if countries take more than a year, they revert back to being "endemic" by definition 12 . The urgency generated by this policy ultimately helps countries develop the response capacity necessary for any infectious disease challenge, mitigating the risk of such challenges becoming threats to the health security of the region, and ultimately to the world.

Examples of recent setbacks include the ongoing measles transmission $>1$-year in Venezuela, and subsequent exportation of this virus to Brazil, with a $>1$-year transmission as a consequence 13 . Obviously, the progress of the regional program digressed with these events. Had measles vaccination coverage been the acceptable 95\% in every district, prolonged transmission would have been prevented. Accurate vaccination coverage is essential for targeting efforts to improve coverage in 
poor performing communities, thus insuring the needed population immunity to prevent widespread transmission after importations.

As a result of its setback, Brazil is trying to strengthen its approach to using data to identify highrisk areas, and to respond accordingly. The challenge is to coordinate efforts among all key actors and beneficiaries, as well as across all levels of government within Brazil. The successful response to the next "Zika-like" intrusion to the health and well-being of newborns will arguably be dependent on how well the current measles situation is handled. Other recent crises in Brazil include Chikungunya, dengue, and yellow fever outbreaks 14. Measles for Brazil and other countries becomes the "litmus test" of the capacity development required for addressing any infectious disease threat, and ultimately global health security.

Colombia is another example of a country facing challenges. Colombia has had multiple different measles virus importations from Venezuela several differente sites within the country (Bogota, Cartagena, Barranquilla, Norte de Santander, and La Guajira), all leading to continuing chains of transmission. The subsequent outbreak responses in each of these locations stopped transmission of imported viruses in Bogota, Cartagena, and Barranquilla. However, it remains to be seen if the responses in Norte de Santander and La Guajira will stop transmission of their imported viruses before the one-year cut-off 13 .

In Barranquilla, the resulting outbreak was fueled by unsafe infectious disease control practices in a large, pediatric, urban hospital of the city. Infected health workers, and triage procedures that exposed other patients unnecessarily to measles infected children, unfortunately amplified transmission. As a result, the hospital conducted a thorough analysis of its infection control procedures that led to dramatic changes in hospital policy. Today, children with fever are screened and triaged to an isolation area, where they remain with families pending their measles laboratory testing results. As a result, patients coming to the hospital for other reasons are not unnecessarily exposed to measles virus. Such changes in policy will also greatly aid future responses to any infectious disease threat. The hospital readily admitted its shortcomings that contributed to outbreak spread, and then immediately acted using data to implement and drive best practices.

\section{Vaccines and antimicrobial resistance}

The most obvious benefit of vaccination in reducing the threat of antimicrobial resistance is the example of widespread administration of pneumococcal conjugate vaccine in countries of the Americas 15. Prevention of invasive pneumococcal disease through vaccination obviates the need for antibiotic therapeutics and the risk of resistance developing. Indirect benefits of childhood vaccination with PCV vaccine include reduction of pneumococcal infection-related mortality in older age groups. The benefit to older adults is almost twice that of the children actually being vaccinated. Other vaccines in the pipeline of development that should provide similar benefits could potentially include new vaccines for malaria, tuberculosis, clostridium infection, Escherichia coli, respiratory syncytial virus, and cholera. All these potential vaccine introductions will require robust surveillance systems to target vaccination and evaluate impact of the strategies. The pipeline of new antibiotics to combat growing resistance is considered by some infectious disease experts as dismally deficient.

To that end, surveillance could be improved by prioritizing a more systematic monitoring of specific resistant pathogens. Reporting those results through appropriate channels within countries, and ideally to regional systems and networks, would ideally enhance the advocacy needed to galvanize more funding for appropriate vaccine research and development. Currently, many experts agree that large gaps exist with systematic monitoring and sharing of information. Coordination across borders is also an important component. 


\section{Summary}

Emerging new pathogens, as well as traditional vaccine preventable diseases, and their consequences, such as increased antimicrobial resistance, challenge national capacities to achieve and maintain effective public health surveillance. Successful response requires using the data to target areas more rapidly where disease is occurring, especially in vulnerable populations, including border populations. Such approaches should help to promote health security across borders.

\section{Additional information}

ORCID: Jon Kim Andrus (0000-0002-8514-8500).

\section{References}

1. Andrus JK, Quadros CA, Ruiz Matus C, Luciani S, Hotez P. New vaccines for developing countries: will it be feast or famine? Am J Law Med 2009; 35:311-22.

2. Andrus JK. Advocating for equity through immunization. In: Berman S, Palfrey JS, Bhutta Z, Grange AO, editors. Global child health advocacy. Washington DC: American Academy of Pediatrics; 2013. p. 73-82.

3. Andrus JK, Castillo Solorzano C, Oliveira L, Danovaro-Holliday MC. Strengthening surveillance: confronting infectious diseases in developing countries. Vaccine 2011; 29 Suppl 4:D126-30.

4. Tambini G, Andrus JK, Fitzsimmons JW, Roses Periago M. Regional programs for health: immunization as a model for strengthening inter-country cooperation and control of infectious diseases. Rev Panam Salud Pública 2006; 20:54-9.

5. Polio Transition Independent Monitoring Board. A debt of honour delivering polio's legacy for those who have suffered and those who have died. Geneva: World Health Organization; 2018. (TIMB's 3rd Report).

6. Andrus JK, Aguilera X, Oliva O, Aldighieri S. Global health security and the international health regulations. BMC Public Health 2010; 10 Suppl:S2.

7. Quadros CA, Andrus JK, Olive J-M, Macedo CG, Henderson DA. Polio eradication from the Western Hemisphere. Annu Rev Public Health 1992; 13:239-52.

8. Andrus JK, Quadros CA, Olive J-M. The surveillance challenge: final stages of eradication of poliomyelitis in the Americas. MMWR CDC Surveill Summ 1992; 41:21-6.
9. Tambini G, Andrus JK, Marques E, Boshell J Quadros CA, Pallansch M, et al. Direct detection of wild poliovirus transmission by stool surveys of healthy children and analysis of community wastewater. J Infect Dis 1993; 168:1510-4.

10. Andrus JK. Leadership in global health: the case of Ciro de Quadros, a testament to values, valor, and vision. Rev Panam Salud Pública 2017; 41:e149.

11. Andrus JK, Quadros CA, Castillo-Solorzano C, Roses Periago M, Henderson DA. Measles and rubella eradication in the Americas. Vaccine 2011; 29 Suppl 4:D91-6.

12. Pan American Health Organization. PAHO Regional Monitoring Commission on Measles Rubella Post-Elimination Meets at PAHO. PAHO Immunization Newsletter 2019; XLI:1.

13. Pan American Health Organization. The challenge of maintaining the elimination of measles in the Americas, 2017-2018. PAHO Immunization Newsletter 2018; XL:1-7.

14. Espinal M, Andrus JK, Jauregui B, Waterman $\mathrm{SH}$, Morens DM, Santos JI, et al. Emerging and reemerging Aedes-transmitted arbovirus infections in the region of the Americas: implications for health policy. Am J Public Health 2019; 109:387-92.

15. Oliveira LH, Toscano CM, Sanwogou NJ, Ruiz Matus C, Tambini G, Roses Periago M, et al. Systematic documentation fo new vaccine introduction in selected countries of Latin America. Vaccine 2013; 31 Suppl 3:C114-22.

Submitted on $01 / \mathrm{Jul} / 2019$

Final version resubmitted on $05 / \mathrm{Feb} / 2020$

Approved on 14/Feb/2020 